\title{
Rate of intracranial self-stimulation as a function of stimulus waveform and intensity'
}

\author{
JIUAN SU, MARK A. BERKLEY, ${ }^{2}$ MICHAEL TERMAN AND J. W. KLING \\ 3ROWN UNIVERSITY
}

Rats bar pressed for two classes of electrical brain stimulation (60) cps sine waves and 100 pps bidirectional rectangular pulses) of varied intensity, presented on VI $15 \mathrm{sec}$. and CRF schedules, under food deprivation and ad lib conditions. The shapes of the rate-intensity functions for the two waveforms appeared similar for individual animals when the stimulus-charge dimension was plotted in log scale. The charge per reinforcement needed with 60 cps was consistently greater than that needed to support equal response rates with 100 pps stimulation, and the range of microcoulombs over which intensities could be varied with 100 pps stimulation was consistently smaller and lower than with $60 \mathrm{cps}$ stimulation.

Several studies on the rate of intracranial selfstimulation have demonstrated the usefulness of amount of electrical charge per reinforcement as a unified expression reflecting the independent variables which determine response rate. Keesey (1962), using bidirectional rectangular pulses, showed that response rate may be expressed as a function of charge, regardless of specific current and frequency parameters. Similarly, McIntire \& Wright (1965), using bidirectional pulse-pairs, noted that high rates are attainable by using either high current intensity with short stimulus duration or low current intensity with relatively long stimulus duration. However, these two studies were not concerned with the question of whether charge consistently determines response rate with different stimulus waveforms, a matter of both theoretical and practical importance for experimental design and comparison. The possibility that differences in waveforms may partially determine efficiency of reinforcement is suggested by the results of Uyeda \& Gengerelli (1959), where sine waves were found to support faster bar pressing than unidirectional rectangular pulses of the same frequency and peak current. However, it was not possible to ascertain if a charge-per-reinforcement analysis would assist in the interpretation of these results. The present experiment was designed to investigate this problem for two frequently utilized waveforms (60 cps sine waves and 100 pps bidirectional rectangular pulses) presented on continuous (CRF) and variable interval (VI $15 \mathrm{sec}$ ) schedules of reinforcement, under food deprivation and ad lib conditions.

\section{Method}

Six male albino rats of the Charles River COBS strain were used. The animals were maintained at $80 \%$ ad lib body weight during the training and deprivation test procedures. Each rat was stereotaxically implanted with a chronic, stainless steel bipolar electrode. All electrodes were aimed at the posterior hypothalamus. Histological verifications of the placements were obtained for three of the animals, showing the electrode tips in the vicinity of the dorsomedial nucleus of the hypothalamus for two of the animals and the ventromedial nucleus for the third.

The 100 pps bidirectional rectangular pulses consisted of alternating positive and negative pulses, each $0.2 \mathrm{msec}$. in duration and equally spaced in time. These were converted from the monophasic output of a Grass Model S-4D rectangular pulse generator by means of a stimulus pulse polarity reversal unit (similar to that described by Alberts, 1958). The $60 \mathrm{cps}$ sine waves were taken from an isolation transformer, stepped down by a variable transformer, and adjusted by a potentiometer. The train duration for both waveforms was held constant at $0.5 \mathrm{sec}$. Stimulus currents were monitored on a cathode ray oscilloscope (peak-to-peak current for $60 \mathrm{cps}$; peak current for 100 pps).

Ss were trained with both waveforms; three started with $100 \mathrm{pps}$, and the other three started with $60 \mathrm{cps}$. Each rat was first trained to bar-press on CRF, using an individually adjusted "medium" stimulus intensity at which it pressed actively, but without any apparent dis ruptive motor involvement. On the second day, "high" and "low" intensities were determined: "high" was set at a level where $S$ occasionally showed squealing and jumping, but yet reliably returned to the bar; "low" was set at a point where the animal maintained a steady, low response rate. Intensity levels for the alternate waveform were obtained in the same manner on the next day. Following CRF training, Ss were trained on the VI $15 \mathrm{sec}$. schedule, alternating between both waveforms for four sessions, 100 reinforcements per session, with intensity set at the medium level. All Ss showed stable responding on both schedules by the end of training.

The test sequence is described in Table 1. Fach animal ran through two test sequences, first under deprivation and then in the ad lib condition, separated by a seven-day weight recovery period. In each daily session, Ss were reinforced at five stimulus intensities, presented in an ascending series of 5-min. intervals. In addition to the three training intensities, intermediate values were derived by taking the arithmetic means of the low and medium, and medium and high intensities, respectively. Response rates were computed on the basis of the last $3 \mathrm{~min}$. of each intensity segment (averaged over the two test days for each waveform), to minimize series effects.

\section{Results and Discussion}

Figure 1 shows the rate of responding as a function of the number of microcoulombs per reinforcement ${ }^{3}$ for all Ss. Response rate functions are similar for the two waveforms, within individual animals, when microcoulombs are plotted in log scale. Maximum and minimum rates were approximately equal for both waveforms under the same schedule (VI or CRF) condition. Although

Table 1. Rate-Intensity Test Sequence

\begin{tabular}{lrrl} 
Session & \multicolumn{2}{c}{ Waveform } & Schedule \\
\hline & Ss $2,5,9$ & Ss $1,6,8$ & \\
1 & $60 \mathrm{cps}$ & $100 \mathrm{pps}$ & VI $15 \mathrm{sec}$. \\
2 & $100 \mathrm{pps}$ & $60 \mathrm{cps}$ & \\
3 & $60 \mathrm{cps}$ & $100 \mathrm{pps}$ & \\
4 & $100 \mathrm{pps}$ & $60 \mathrm{cps}$ & \\
5 & $60 \mathrm{cps}$ & $100 \mathrm{pps}$ & CRF \\
6 & $100 \mathrm{pps}$ & $60 \mathrm{cps}$ & \\
7 & $60 \mathrm{cps}$ & $100 \mathrm{pps}$ & \\
8 & $100 \mathrm{pps}$ & $60 \mathrm{cps}$ & \\
\hline
\end{tabular}




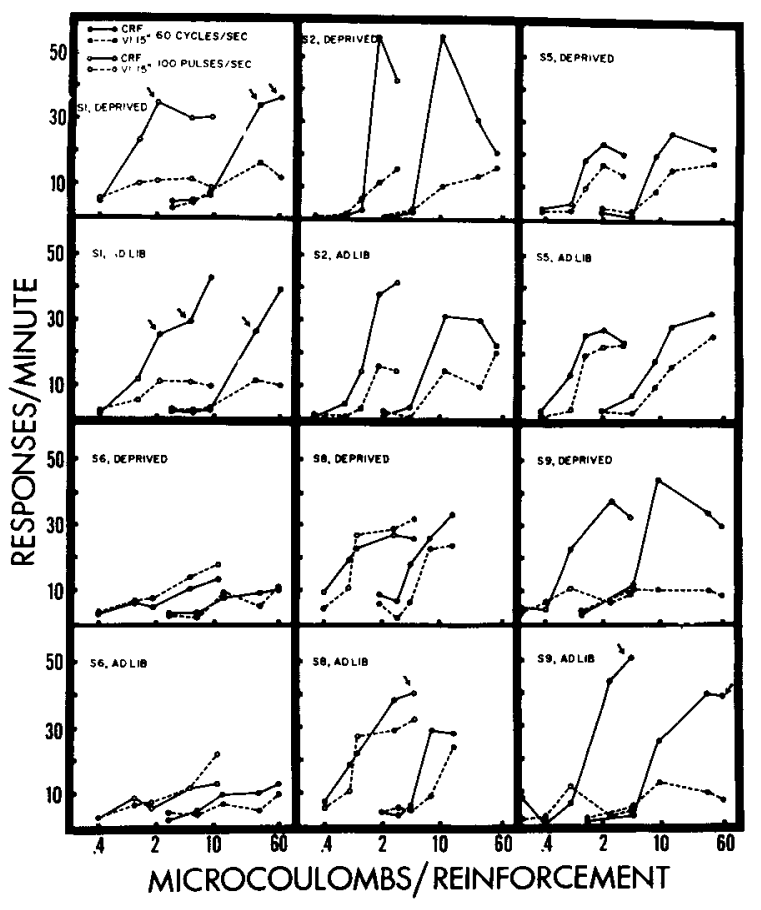

Fig. 1. Rate of responding as a function of the microcoulombs per reinforcement. Arrows indicate occurrence of occasional disruptive motor effects. In each box, the curves on the left are 100 pps functions; those on the right, 60 cps functions.

the effective ranges of charge per reinforcement for each waveform appear to be displaced by a constant, they cover approximately equivalent numbers of log units. For every animal, the charge per reinforcement needed with $60 \mathrm{cps}$ was almost always greater than that needed to obtain equal response rates with $100 \mathrm{pps}$ stimulation. In regions where the curves overlap on the stimulus-charge dimension, direct comparison of rates resulting from equal charge per reinforcement values indicates that 100 pps stimulation consistently yielded higher response rates. The only exception occurred in slow VI 15 sec. responding by S9. Stimulation by 100 pps bidirectional rectangular pulses may be characterized as more "efficient" than stimulation by $60 \mathrm{cps}$ sine waves, in terms of the electrical charge per reinforcement required to support equal response rates. The physical differences between the waveforms which may be determinants of relative efficiency merit future investigation.

The general effect of increasing intensity was to increase response rate on both CRF and VI $15 \mathrm{sec}_{0}$, but some of these functions are non-monotonic, a fact attributed to the temporal density of stimulations by
Beer et al (1965). No systematic differences are apparent between the data obtained under food deprivation and ad lib conditions.

The value of charge per reinforcement as an integrative device has been criticized by Ridgway et al (1965), who reported that the reinforcing effects of 500 cps sine wave stimulation varied as a joint function of train duration and stimulus intensity, even though charge per reinforcement was held constant. ${ }^{4}$ However, their data may not be incompatible with the charge interpretation, if variations in train duration are found to modify the rate-charge relation by some constant factor. Indeed, if Keesey's (1964, p. 206) response rate data are expressed in terms of log charge per reinforcement, the rate intensity functions do appear to be displaced by a constant, for each train duration. In analogy to the present experiment, Keesey's data indicate that shorter train durations were more "efficient" for supporting equal response rates than longer ones. Thus, the available parametric data suggest a rewording of the "charge hypothesis": for any given waveform or train duration, response rate will be a function of log charge per reinforcement as intensity is varied, while changes in waveform or train duration will require a general adjustment in charge per reinforcement, due to differing effective ranges.

\section{References}

Alberts, W. W. A stimulus pulse polarity reversal unit. EEG clin. Neurophysiol., 1958, 10, 172-173.

Beer, B., Hodos, W., \& Matthews, T. J. Rate of intracranial selfstimulation as a function of reinforcement magnitude and density. Psychon. Sci., 1964, 1, 321-322.

Keesey, R. E. The relation between pulse frequency, intensity and duration and the rate of responding for intracranial stimulation. J. comp. physiol. Psychol., 1962, 55, 671-678.

Keesey, R. E. Duration of stimulation and the reward properties of hypothalamic stimulation. J. comp. physiol. Psychol., 1964. 58, 201-207.

McIntire, R. W., \& Wright, J. E. Parameters related to response rate for septal and medial forebrain bundle stimulation. $J$. comp. physiol. Psychol., 1965, 59, 131-134.

Ridgway, J. E., Pliskoff, S. S., \& McIntire, R. W. The utility of the microcoulomb as a predictor of reinforcement value in electrical stimulation of the rat brain. Psychon. Sci., 1965, 3, 109110 .

Uyeda, A. H., \& Gengerelli, J. A. Influences of rectangular pulses and sine waves of varying frequencies on brain self-stimulation in the laboratory rat. Psychol. Rep., 1959, 5, 641-647.

\section{lotes}

1. Supported by USPHS Grant No. MH-05767.

2. Now at Department of Biophysics and Physiology, University of Washington.

3 . Computed from average current for $60 \mathrm{cps}$ and peak current for $100 \mathrm{pps}$.

4. At $20 \mathrm{cps}$ and $2000 \mathrm{cps}$, the same value of charge per reinforcement used at $500 \mathrm{cps}$ appeared to be non-reinforcing. 